\title{
Space and momentum representation analysis of Hartman's effect in wave packet transmission
}

\author{
María F. González, ${ }^{1,2}$ Josep Maria Bofill, ${ }^{3,2}$ Xavier Giménez, ${ }^{1,2}$ and F. Borondo ${ }^{4}$ \\ ${ }^{1}$ Departament de Química Física, Universitat de Barcelona, Martí i Franquès 1, 08028 Barcelona, Spain \\ ${ }^{2}$ Centre Especial de Recerca en Química Teòrica, Universitat de Barcelona, Baldiri Reixach 10-12, 08028 Barcelona, Spain \\ ${ }^{3}$ Departament de Química Orgànica, Universitat de Barcelona, Martí i Franquès 1, 08028 Barcelona, Spain \\ ${ }^{4}$ Departamento de Química, Universidad Autónoma de Madrid, and Instituto de Ciencias Matemáticas CSIC-UAM-UC3M-UCM, \\ Cantoblanco, 28049 Madrid, Spain
}

(Received 25 February 2008; revised manuscript received 22 July 2008; published 3 September 2008)

\begin{abstract}
The Hartman effect is analyzed in both the position and momentum representations of the problem. The importance of Wigner tunneling and deep tunneling is singled out. It is shown quantitatively how the barrier acts as a filter for low momenta (quantum speed up) as the width increases, and a detailed mechanism is proposed. Superluminal transmission is also discussed.
\end{abstract}

DOI: 10.1103/PhysRevA.78.032102

PACS number(s): 03.65.Xp, 03.75.Lm, 42.50.Xa

\section{INTRODUCTION}

Not surprisingly, quantum wave packet dynamics show distinctive features when compared to classical dynamics. However, they also show intriguing peculiarities when faced against seemingly equivalent time-independent quantum treatments. One of them is the so-called Hartman effect (HE) [1], namely, the independence of the tunneling time with the value of the barrier width. This effect is thought to be of general validity and takes place in a wide variety of scattering conditions [2]. The mechanism is attributed to the specific role of the plane wave components which combine in the transmitted wave defining a larger propagation velocity. In the words of Landauer and Martin [3], the barrier acts as a wave packet accelerator with respect to the propagation velocity before collision. Several authors have focused their attention on HE $[4,5]$, including extensions to take into account wave packet time spreading [6], multiple barriers [7], wells [8], absorbing potentials [9], quantum networks [10], and even the negative HE [11]. Most of these works are based on analysis relying in plane waves. Recent interpretations resort to a saturation in the penetration depth of the tunneled wave function for sufficiently wide barriers [12]. However, Winful [13] has considered a complementary view in terms of energy storage of electromagnetic waves [14,15]; this allowed us to question some previous results [7] concerning superluminality. HE takes place due to a reshaping mechanism similar to that in weak quantum measurements, and causality prevents supervelocities for a potential not supporting bound states [16]. Very recently, Chen and Liu [17] discussed superluminal transversal times in terms of interference between multiple finite wave packets.

This controversy and others [18] points towards the need for complementary interpretational views of HE, which is the purpose of the present paper. We use the momentum as a primary magnitude, thus obtaining a better insight for the HE, that is important in many atomic and molecular applications.

The combined use of position and momentum representations also proves instrumental to focus on tunnel "tagging" or classification within HE. In this respect, two main regimes, direct and deep tunneling $[19,20]$, should be distin- guished. See, for example, Heller [21] for outstanding examples in dynamics, spectroscopy, and photochemistry. Transmission for those energies lying above the barrier is called direct or Wigner (since this is frequently associated to classical trajectories starting from a Wigner quantum mechanical distribution in phase space) tunneling. Other authors regard this as shallow tunneling [22]. On the other hand, energy components of a wave packet lying below the energy barrier give rise to transmission by deep tunneling, a process which connects classically disjoint regions of phase space $[20,22,23]$. This is important for low temperatures, high electronic energy barriers, weak intersite electronic coupling, off resonance, or breakdown of the Franck-Condon approximation [24]. It has also been of interest in semiclassical mechanics, where it has been treated both with real and complex-valued quantities [23]. Finally, we would like to point out that other wave packet studies are available thanks to recent advances in femtosecond physics [25].

The organization of the paper is as follows. In Sec. II we focus on the theoretical tools used in our study. Some results are then presented and discussed in Sec. III. Finally, in Sec. IV we summarize our main conclusions and ideas for future developments.

\section{THEORY}

The problem considered in this work is that of a minimum uncertainty Gaussian wave packet set to collide against a potential barrier. In the first part of the paper, the central (maximum) momentum value and its width parameter are selected so that no appreciable fraction of the wave packet has a significant momentum above that corresponding to the maximum of the potential barrier. Accordingly, the transmission is a purely quantum mechanical process, and we are considering essentially deep tunneling events. In the second part, the value of the momentum is increased, so that the amount of Wigner or direct tunneling grows. In this way, we get a better characterization of the transmitted particle quantum speed up, as well as the intensity of the transmitted packet. The role of both Wigner and deep tunneling events will be described in detail. 
The propagation algorithm that will be used has been described elsewhere [26,27]; we then give only a brief description of it to make the paper self-contained. The packet is described in terms of a suitable basis set as

$$
\psi(x, t)=\sum_{j=1}^{N} \phi_{j}(x)\left\langle\phi_{j} \mid \varphi_{0}\right\rangle e^{-i E_{j} t / \hbar},
$$

where just one spatial dimension has been indicated, being the extension straightforward. The initial wave packet, $\varphi_{0}(x)$, is chosen as a harmonic coherent-state (atomic units will be used throughout the paper)

$$
\varphi_{0}(x)=(2 \alpha / \pi)^{1 / 4} \exp \left[-\alpha\left(x-x_{0}\right)^{2}+i p_{0}\left(x-x_{0}\right)\right],
$$

giving $\left(x_{0}, p_{0}\right)$ the position of the center of the packet and $\alpha$ is related to its width. The basis set we used consists of the (orthogonal) primitive functions diagonalizing the position operator $\hat{x}$ with equally spaced eigenvalues, i.e., a set of eigenfunctions $\hat{x}\left|x_{i}\right\rangle=x_{i}\left|x_{i}\right\rangle, \quad\left\langle x_{i} \mid x_{j}\right\rangle=\delta_{i j}$, where $\left\{\left|x_{i}\right\rangle\right\}, \quad i$ $=1, \ldots, N$ are Dirac-delta functions in the position representation. This requires starting from a sinc discrete variable representation (DVR) original basis prior to diagonalizing the position operator. This procedure leads to an especially simple propagation algorithm [26], which in matrix form can be written as

$$
\mathbf{y}(t)=\mathbf{L}^{T} \cdot \mathbf{T} \cdot \mathbf{L} \cdot \mathbf{j}_{0},
$$

where $\mathbf{y}(t)$ is the $N$-vector giving the total wave function at the grid points at time $t, \mathbf{T}$ is the diagonal matrix with elements $e^{-i E_{j} t}, j=1, \ldots, N, \mathbf{L}$ is the eigenvectors matrix associated to the DVR-stationary basis change, and $\mathbf{j}_{0}$ is the vector corresponding to the initial wave packet, with components corresponding to its value at each grid position. Matrices $\mathbf{j}_{0}$, $\mathbf{L}$, and $\mathbf{L}^{T}$ matrices are computed only once, prior to start the time propagation. Consequently, any step in the time propagation amounts to updating matrix $\mathbf{T}$ according to the new value for the time variable. Thus, $\mathbf{y}(t)$ is obtained by simple matrix multiplication. Convergence is ensured by repeating the calculation with an increasing number of primitive basis functions (equivalent to reducing the grid increment $\Delta x$ ), until results become stable.

In the present paper we also use the momentum representation. This can be easily achieved in our case by Fourier transformation

$$
\mathbf{y}_{p}(t)=\mathbf{F} \cdot \mathbf{L}^{T} \cdot \mathbf{t} \cdot \mathbf{L} \cdot \mathbf{y}_{0},
$$

where $\mathbf{y}_{p}(t)$ is the wave function amplitude in the momentum space, $\mathbf{F}$ is the Fourier transform matrix,

$$
F_{m n}=\Delta x \exp \left(-i x_{m} p_{n}\right),
$$

where $p_{n}$ is the $n$th discrete momentum. [Notice that Eq. (5) arises from a simple rectangular algorithm for the numerical integration of Fourier transforms.] Numerical convergence, in both position and momentum, is controlled by simply changing the position space increment $\Delta x$.

\section{RESULTS AND DISCUSSION}

A wave packet with $\left(x_{0}, p_{0}\right)=(-1.5,7)$ hitting a square barrier located between $x=0$ and $x=B W$ with a height of

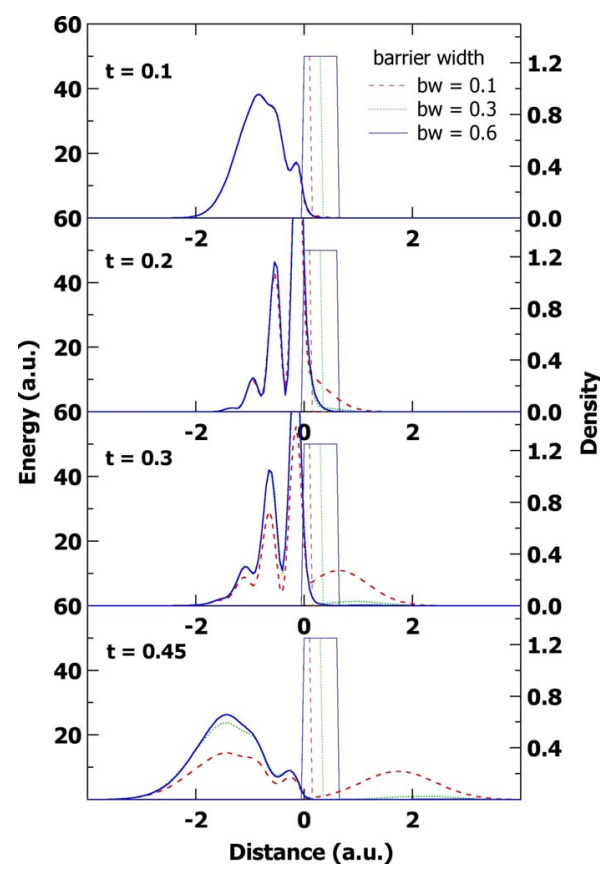

FIG. 1. (Color online) Four different time snapshots describing the wave packet passage across a square barrier. Three different values of the barrier width $B W=0.1$ (red dashed line), 0.3 (green dotted line), and 0.6 (blue full line) are considered.

$V_{0}=50$ (which corresponds to $10 \mathrm{a}$.u. in momentum units) is considered first. Figure 1 shows some time snapshots of the transmission across barriers of different widths, in position space. The time snapshots corresponding to the three barrier widths have been plotted superimposed, so as to show the differences existing in the transmission process, as $B W$ gets larger. At the beginning $(t=0.1)$ no differences in the wave packets motion are observed. Afterwards $(t=0.2$ and 0.3$)$, the initial stages of the overlap between the wave packet with the barrier begin, and then again no important differences among the three cases are found. One feature is worth stressing, however. The reflected part of the wave packet shows the same interference pattern for the three cases. Surprisingly, all maxima and minima describing interference appear located at the same positions, the only difference being that the corresponding intensities are larger as $B W$ increases. It is clear that the barrier as a whole, not just the left wall, influences the reflected part of the packet. Moreover, this takes place in a very interesting way. The transmitted intensity, which decreases as $B W$ increases, makes the corresponding reflected wave more intense. However, the reflected wave appears to "come back" in a time interval which is independent of the value of $B W$. The corresponding modulus, however, grows with this parameter. In other words, a change in the barrier width has the only effect of changing the intensity of the maxima and minima, but not their general structure, i.e., their location in position space, as a function of time. For longer times $(t=0.4)$ the transmitted part of the three packets considered develops. Their intensities and the probability areas they define with the horizontal axis decreases with $B W$. More interestingly, it is observed that the position of the maximum of the transmitted packet shifts toward 


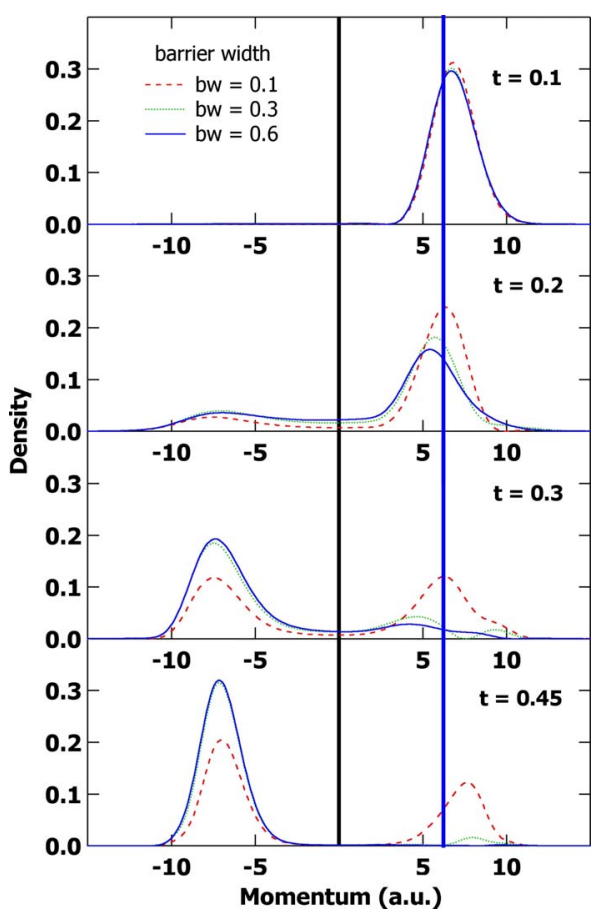

FIG. 2. (Color online) Same as Fig. 1 but in momentum representation. The vertical blue trace corresponds to the barrier height location in momentum units.

larger values, at equal times, the larger $B W$ is. These two joint characteristics, namely, the simultaneous decrease in the transmitted area and the peak shifting towards larger positions, may be regarded as the position representation version of the HE. This shows the advantage of considering the complete wave packet time evolution, instead of just discussing the individual plane-wave components, when analyzing this type of phenomena.

Figure 2 is analogous to Fig. 1 but in the momentum representation. Accordingly, it consists of a series of snapshots describing the wave packet transmission in momentum coordinates. The first picture $(t=0.1)$ depicts the initial stages of the process, where the packet (momentum distribution) changes only very slightly. For $t=0.2$ (second snapshot) the wave packet begins to be distorted, evidencing the wellknown wiggles due to interference between the positive and negative momentum components of the wave packet. This effect is clearly seen in our momentum space picture, where it is found that the negative momenta develop a rather small area, compared to the wave packet intensity at the left of the barrier in the position representation (compare with the equivalent snapshot in Fig. 1). We are then led to conclude that this part of the packet contains both positive and negative momenta, i.e., components of the packet traveling forward and backward. In the third snapshot $(t=0.3)$ the collision with the barrier has progressed to a large extent. Negative momenta span now a more substantial area than the positive momenta, indicating that the reflection is close to being completed. Interestingly enough is that the region corresponding to positive momenta shows a bimodal distribution for the values of $B W$ considered, with the areas decreasing as the barrier width gets larger. This is the low-energy equivalent of the Brouard-Muga effect [28], previously shown for a packet which experiences transmission over a small potential energy barrier. Finally, the last snapshot $(t=0.45)$ shows the situation when the transmission is complete. The overall (positive plus negative) momentum distribution is clearly bimodal, being close to a Gaussian in both negative and positive momenta sections. The positive momenta displays a dramatic decrease in the area as $B W$ gets larger. This is the momentum representation version of the HE. Before moving on, it is worth considering how the central momentum of both reflected and transmitted parts of the wave packet changes as a function of time. At early times in the transmission process, the central momentum of the reflected part of the packet decreases with time. This clearly shows that the slowest components of the reflected part starts to contribute much later to the momentum distribution. On the other hand, the central momentum of the transmitted part behaves completely different. At the earliest times the maximum appears at lower values of the momentum. At intermediate times a bimodal distribution develops, the first maximum being lower, whereas the second maximum appears at much larger values. Finally, late times display the survival, in the transmitted packet, of just the right maximum of the original bimodal distribution, the left one having been transferred to the reflected part of the packet. This effect can be interpreted as a quantum slowdown.

Let us consider now the time dependence of the momentum distributions that we have just presented. Actually, what we are seeking is to explain the differences among the results obtained for the different values of the barrier width that we have used. For this purpose we will make use of the wave functions time derivative as a function of the elapsed time. Figure 3 shows such results in momentum space for two different values of $B W=0.1$ and 0.3 . Fewer values of this parameter are considered now for the sake of clarity, although the conclusions drawn are of general validity. The wave function time derivative gives a clear indication on non-negligible momenta experiencing a substantial change in amplitude, as a consequence of the dynamical process. In particular, our results for the time dependence clearly indicates the presence of two effects. First, the transfer from positive to negative momentum values is mediated by a sudden pulse, which propagates backwards (from positive to negative momenta). In addition, this pulse is more intense for larger values of the barrier width. Even when this difference in intensity is not too big, it is very persistent in time. The second remarkable effect is the existence of a radiatinglike backwards change in momentum amplitude for large values of the momentum. This radiation takes place when the wave packet reaches the end of the square barrier, i.e., when it collides with the barrier right edge. Consequently, this radiation appears at later times for larger values of $B W$.

Figure 4 shows the transmission probability as a function of the barrier width, for several values of the central incident wave packet momentum. These results provide a quantification of the amount of transmission, based on complete numerical wave packet calculations. Moreover, they put in the right context the likelihood of "superluminal" transmission for massive particles (albeit on a pure nonrelativistic context), since they explicitly show that, above a given value of 

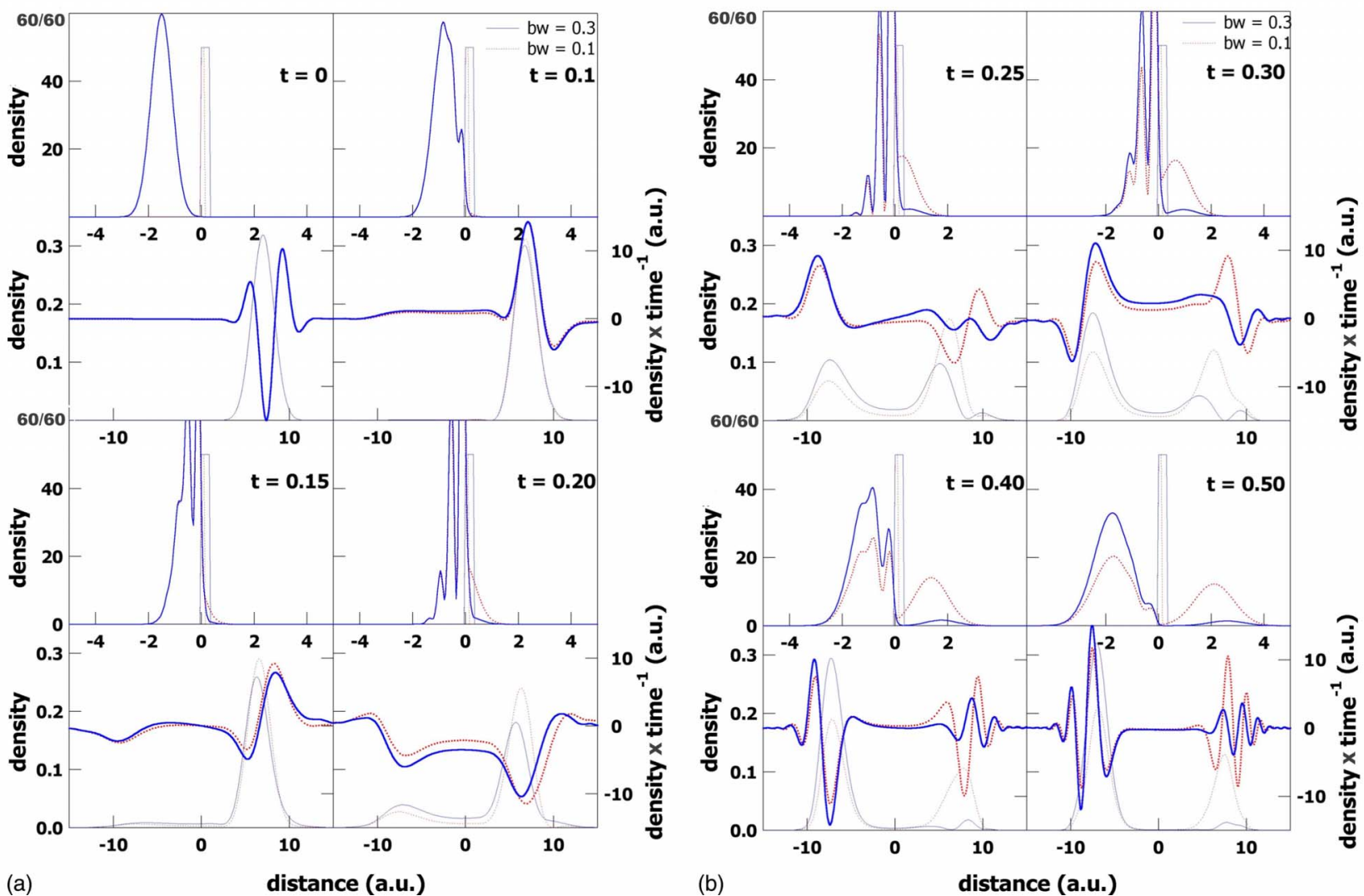

FIG. 3. (Color online) Upper panels: Time snapshots for the density. Lower panels: Time snapshots for the momentum density (thin lines) and the momentum density time derivative (thick panels). Two values of the barrier width have been selected. WB=0.1 (red dotted lines) and 0.3 (blue solid lines).

$B W$, the true numerically converged transmission amplitude is so small that virtually no particles emerge at the right part of the barrier. In other words, the price to pay to get superluminality is that the intensity of the packet is so much depleted that no transmitted particles are able to propagate that fast. However, Fig. 4 provides some useful additional information. In it we show with a solid line the results corresponding to the actual full quantum calculations, with a dotted line the Wigner or direct transmission probability, i.e., the area at large times lying at the right of the barrier height momentum space, and with a dashed line the results corresponding to the $t=0$ area located to the right of the barrier height in the momentum representation, i.e., the classical transmission of a momentum distribution classically mimicking the initial quantum wave packet. This classical, "right" transmission probability is found to be much larger than both the full quantum and the Wigner ones, thus showing that antitunneling plays a major role in the process. This is by no means counteracted by deep tunneling. Whereas this deep tunneling may be rigorously calculated as the infinite time area for positive momenta lying below the barrier height (see the $t=0.45$ case of Fig. 2), it also corresponds, in the present case, to the difference between Wigner and actual transmission cases. Hence, deep tunneling is larger the smaller is the barrier width, whereas it becomes negligible for sufficiently wide barriers. Then, it may be concluded that an increase in the barrier width depletes only the deep tunneling events.
Figure 5 shows results for the central momentum of the transmitted density as a function of the barrier width, for several values of the central incident wave packet momentum. Two main conclusions can be drawn from this figure. First, it is noticed that the transmitted momentum gets larger as the barrier width is increased for those initial momenta having a sufficiently large contribution from deep tunneling momenta. Second, it is observed that this central momentum tends toward a nearly constant value for very large barrier widths, irrespective of the initial central momentum of the wave packet. The reason for such behavior can be found in the nearly negligible tail of the wave packet, allowing for direct tunneling transmission. As the barrier gets wider, the momentum components transmitting through deep tunneling get essentially deployed, so that only those components of the initial wave packet, transmitting by means of direct tunneling, do lead to a transmitted packet. Moreover, it is found that the maximum speedup, i.e., the maximum shown by every trace in Fig. 5, approximately corresponds to the barrier height. Actually, this maximum value is higher than the barrier height (10 a.u. in our case), but it gets lower as the lower incident central momentum is decreased. Consequently, it appears as if the maximum speedup represents an upper bound to the barrier height, this bound being lower for lower values of the incident central momentum. One may reverse the above argument, and state that it is (approximately) possible to infer the barrier height from speedup 


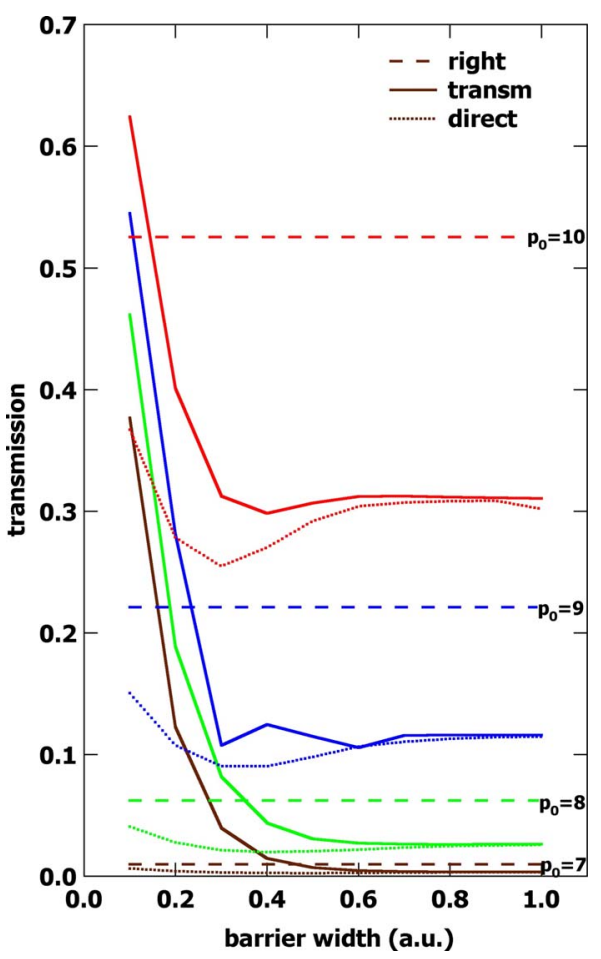

FIG. 4. (Color online) Transmission probability as a function of the barrier width for several values of the wave packet central momentum (indicated in the graph): (solid line) full quantum calculation, (dotted line) results for the momenta above the barrier height, (dashed line) area to the right to the barrier height in momentum representation for $t=0$, thus corresponding to the classical transmission.

measurements for those systems in which it is possible to tune the barrier width. This may be an possible alternative to the search for inflexion points in excitation function measurements.

Figure 6 is similar to Fig. 2 above but for the Eckart barrier [29]. Three different values of the widths have been

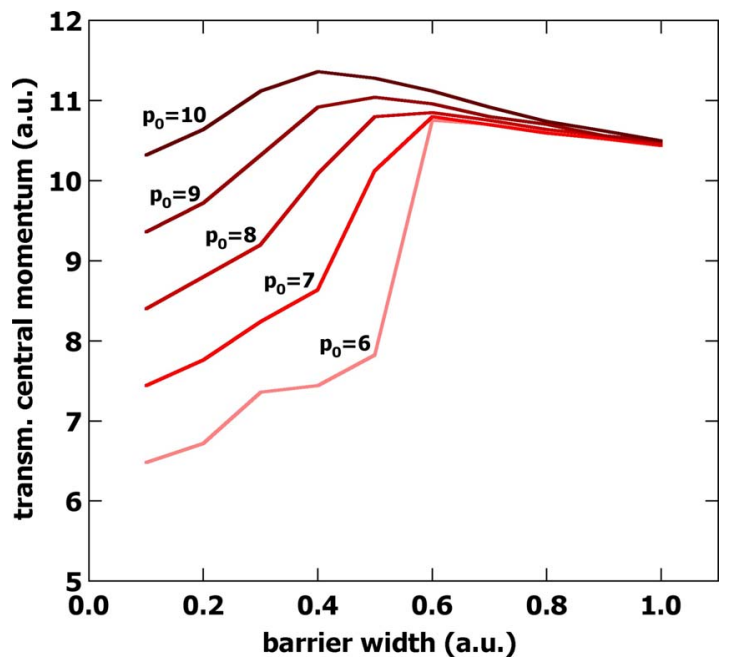

FIG. 5. (Color online) Central (most probable) momentum of the transmitted wave packet as a function of the barrier width, for several values of the initial central momentum of the packet $p_{0}=6$, $7,8,9$, and 10 .

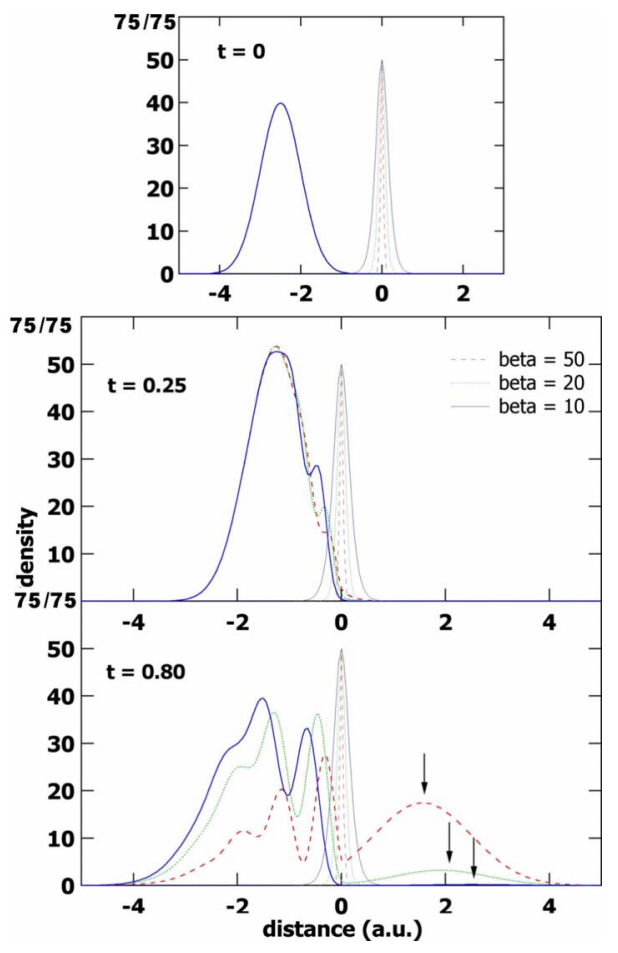

FIG. 6. (Color online) Same as Fig. 3 for three Eckart barriers of increasing width. The arrows indicate the position of the maxima, in order to evidence their shifting to larger positions for larger barrier widths.

considered, chosen in such a way that the potential maxima are located at the same position. The interest of this study, in which a different type of potential is used, is to determine whether the HE arises as a consequence of the specific features of the square barrier, or rather the filtering effect for tunneling momenta is a much more general feature. Actually, since for the Eckart case the barrier height is not constant, it would be surprising to find different outcomes for the HE. Results in Fig. 6 show that this is not the case. Rather, it is observed that the existence of a filtering effect similar to that found for the square barrier, in which wider barriers lead to faster transmitted wave packets. The only difference being that for the Eckart barrier the frequency of the reflected wiggles is lower. Consequently, one may state that the $\mathrm{HE}$ is not a phenomenon specific of square barriers, and then it should be expected in general potential profiles. This opens the possibility of extrapolating the consequences of the $\mathrm{HE}$, in either time, position or momentum domains, to the realm of atomic and molecular physics, including chemical reactions.

\section{SUMMARY AND CONCLUSIONS}

Summarizing, we have presented a theoretical study of the HE, providing a complementary view to those contained in previous literature. For this purpose, we have used both the position and momentum space representations, in order to have a better understanding of the underlying dynamics. The second approach has shown to be very informative, since it allows a clear-cut distinction between the direct and 
deep tunneling events, and also to propose (through consideration of the wave function time derivative) the detailed mechanism by which quantum transmission decreases as the barrier width gets larger.

The present view suggest several alternative possible experimental measurements involving tunneling. First, a comparison between incident and transmitted central momenta should evidence a quantum speed up proportional to the traversed barrier width. To perform this measurement it is necessary to: (1) an initial coherent state showing typical widths (in both position and momentum) comparable to the size of the barrier, (2) an initial mean momentum located well below the barrier height so that tunneling is dominant, (3) a light particle (to maximize tunneling), and (4) a detector for the transmitted products with enough velocity (momentum) resolution able to measure the quantum speed up. Second, the quantum speed up of the transmitted particle may be reversed. Measuring its amount of speed up one should be able to infer the barrier width, after a straightforward deconvolu- tion procedure. Since information on the barrier height is frequently available from scattering excitation function measurements, the present proposal provides a simple prospective method to increase the overall experimental knowledge of suitable physical process. Third, the existence of a quantum speed up on the transmitted particle ( $p$-space version of the $\mathrm{HE}$ ) might have an influence in reaction rates of complex processes. This effect is normally not taken into account in the usual plane wave treatments.

\section{ACKNOWLEDGMENTS}

We have benefited from discussions with Professor Muga. Financial support from MCyT (Spain) under Contract Nos. CTQ2005-01117-BQU, MTM2006-15533, and CONSOLIDER 2006-32, Generalitat de Catalunya Projects Nos. 2005SGR-00111 and 2005SGR-00175, and Comunidad de Madrid Project No. S-0505/ESP-0158, is acknowledged.
[1] T. E. Hartman, J. Appl. Phys. 33, 3427 (1962).

[2] J. G. Muga and C. R. Leavens, Phys. Rep. 338, 353 (2000).

[3] R. Landauer and T. Martin, Rev. Mod. Phys. 66, 217 (1994).

[4] J. Weis and O. Weis, Eur. Phys. J. B 12, 135 (1999).

[5] M. Sassoli de Bianchi, Eur. J. Phys. 21, L21 (2000).

[6] V. S. Olkhovsky, V. Petrillo, and A. K. Zaichenko, Phys. Rev. A 70, 034103 (2004).

[7] S. Esposito, Phys. Rev. E 67, 016609 (2003).

[8] J. G. Muga, I. L. Egusquiza, J. A. Damborenea, and F. Delgado, Phys. Rev. A 66, 042115 (2002).

[9] F. Delgado, J. G. Muga, and A. Ruschhaupt, Phys. Rev. A 69, 022106 (2004).

[10] S. Bandopadhyay and A. M. Jayannavar, Phys. Lett. A 335, 266 (2005).

[11] L.-G. Wang, J.-P. Xu, and S.-Y. Zhu, Phys. Rev. E 70, 066624 (2004).

[12] J. C. Martínez and E. Polatdemir, Phys. Lett. A 351, 31 (2006).

[13] H. G. Winful, Phys. Rev. Lett. 90, 023901 (2003); 91, 260401 (2003); Nature (London) 424, 638 (2003); Phys. Rev. E 68, 016615 (2003).

[14] T. Martin and R. Landauer, Phys. Rev. A 45, 2611 (1992).

[15] S. Zhu, A. W. Yu, D. Hawley, and R. Roy, Am. J. Phys. 54, 601 (1986).
[16] D. Sokolovski, A. Z. Msezane, and V. R. Shaginyan, Phys. Rev. A 71, 064103 (2005); 76, 042125 (2007).

[17] X. Chen and C.-F. Li, Europhys. Lett. 82, 30009 (2008).

[18] M. Büttiker and S. Washburn, Nature (London) 422, 271 (2003); 424, 638 (2003).

[19] D. H. Zhang and E. Pollak, Phys. Rev. Lett. 93, 140401 (2004)

[20] N. T. Maitra and E. J. Heller, Phys. Rev. Lett. 78, 3035 (1997).

[21] E. J. Heller, J. Phys. Chem. A 103, 10433 (1999).

[22] K. G. Kay, J. Chem. Phys. 107, 2313 (1997).

[23] S. Keshavamurthy and W. H. Miller, Chem. Phys. Lett. 218 , 189 (1994).

[24] M. Caspary and U. Peskin, J. Chem. Phys. 123, 151101 (2005).

[25] S. A. Rice and M. Zhao, Optical Control of Molecular Dynamics (Wiley, New York, 2000).

[26] J. González, J. M. Bofill, and X. Giménez, J. Chem. Phys. 120, 10961 (2004).

[27] J. González, M. F. González, J. M. Bofill, and X. Giménez, J. Mol. Struct.: THEOCHEM 727, 205 (2005).

[28] A. L. Pérez Prieto, S. Brouard, and J. G. Muga, Phys. Rev. A 71, 012703 (2005).

[29] C. Eckart, Phys. Rev. 35, 1303 (1930). 